\title{
Early Lesion of Post-Primary Tuberculosis: Subclinical Driver of Disease and Target for Vaccines and Host-directed Therapies
}

\author{
Robert E. Brown ${ }^{1}$, Robert L. Hunter ${ }^{1, *}$ \\ 1Department of Pathology and Laboratory Medicine, University of Texas Health Sciences Center at Hou-ston, \\ Houston, TX 77030, USA: robert.brown@uth.tmc.edu, Robert.L.Hunter@uth.tmc.edu \\ * Correspondence: Robert.L.Hunter@uth.tmc.edu Tel: 001713898 0666d
}

\begin{abstract}
Research on the pathogenesis of tuberculosis in recent years has focused largely on the granulomatous stage of primary tuberculosis. However, post-primary tuberculosis that accounts for $80 \%$ of clinical disease is seldom studied because of the paucity of animal models and human tissues. The early lesion of post-primary tubercu-losis is a subclinical obstructive lobular pneumonia that develops asymptomatically for months accumulating secreted mycobacterial antigens in alveolar macrophages and highly sensitized $\mathrm{T}$ cells before onset of clinical disease. Here we demonstrate antigen of M. tuberculosis in the cytoplasm of ciliated bronchiolar epithelium and alveolar pneumocytes in association with elements of the programmed death ligand 1 (PD-L1), cyclo-oxygenase (COX)-2, and fatty acid synthase (FAS) pathways in the early lesion. This suggests a new synthesis of the pathogenesis of post-primary tuberculosis in which $\mathrm{M}$. tuberculosis use its secreted antigens and cord factor to direct prolonged subclinical development of the early lesions in preparation for a sudden necrotizing reaction sufficient to produce a cavity and/or granulomas. Available evidence indicates that most successful human and animal vaccines and host directed therapies of post-primary tuberculosis target the early lesion, not granulomas. Recognition of this will facilitate design and evaluation of improved vaccines and therapies for tuberculosis.
\end{abstract}

Keywords: tuberculosis, secretory antigens, bronchiolar epithelium, alveolar pneumocytes, M2 polarization, COX-2, FAS. Pathogenesis, early lesion

\section{Introduction}

Pathologists in the pre antibiotic era were familiar with a series of tuberculous lesions that are seldom seen to-day [1]. They recognized that the onset of clinical post-primary tuberculosis (PPTB) is preceded by 1-2 years asymptomatic subclinical development of the early lesion in the lung before appearance of caseous pneumonia and granulomas [2-4]. Today, since there is no medical reason to biopsy such lesions and autopsies are infre-quent, most investigators are unaware of their existence even though they remain familiar to radiologists as the 'tree-in-bud' sign characteristic of developing PPTB [5].

The early lesions of PPTB do not begin until after establishment of sufficient immunity to prevent disease in all extra pulmonary and most pulmonary sites [1]. PPTB begins as an alveolitis and spreads for months via bronchi as an obstructive lobular pneumonia that is recognizable on high resolution CT scans as the 'tree-in-bud' sign [5]. Foamy alveolar macrophages accumulate behind obstructed bronchi in close association with highly sensitized T cells. Many lesions spontaneously regress, but some undergo necrosis to produce caseous pneumonia that is either coughed out to form cavities or is retained to become the focus of post-primary granu-lomas and fibrocaseous disease [6]. Post-primary granulomas are easily identified because they contain ghosts of alveoli rather than the homogeneous caseum of primary granulomas [1]. Primary and post-primary granulo-mas are seldom found together in the same lung. We reported sequestration of secreted mycobacterial antigens in foamy alveolar macrophages in the 
developing lesions of post-primary TB and that they are released with the onset of caseation necrosis in a fashion suggestive of the Koch phenomenon [6].

Here we report finding M. tuberculosis (MTB) antigens, but not acid fast bacilli (AFB), in alveolar lining cells (pneumocytes) and ciliated bronchial cells in addition to foamy alveolar macrophages in developing lesions of PPTB. This suggested that MTB use secreted antigens and cord factor to orchestrate an extended sequence of subclinical lesions that prepare for a sudden massive necrotizing reaction sufficient to produce cavities from which the organisms can escape to new hosts. Post-primary granulomas arise to surround foci of caseous pneumonia that are not coughed out to form cavities. They persist to become fibrocaseous disease. Available evidence suggests that several components of the early lesion of PPTB are attractive targets for vaccines and/or host-directed therapies. Most nascent lesions regress spontaneously leaving apical scars. If we understood why they regress, it might be possible to make them all regress and thereby drive MTB to extinction.

\section{Material and Methods}

Formalin-fixed and paraffin-embedded tissue blocks of over 100 cases of human tuberculous lung samples were obtained during regular autopsy practice and after the completion of all medical, legal and ethical re-quirements and were de-identified. Furthermore, this study was conducted according to the principles ex-pressed in the Declaration of Helsinki. Hematoxylin and eosin (H\&E) staining was used to identify cases that of the early lesion of post-primary TB [1].

Immunohistochemistry was performed on five micrometer sections that were deparaffinized and stained with monoclonal antibodies for: MTB antigen (ab905, Abcam, Cambridge, MA): cyclo-oxygenase (COX)-2 (SP21rabbit, Biocare Medical, Pacheco, CA); Programmed Death-Ligand 1 (PD-L1: Spring Bioscience, Pleasanton, CA); Programmed Death-1 (PD-1, Biocare Medical); and fatty acid synthase (FAS:C20G5, Cell Signaling Technology). Positive and negative controls were run concurrently. The immunohistochemical reac-tions/ expressions were graded in accordance with the brown chromogenic signal intensity of the 3,3'-diaminobenzidine (DAB).

\section{Results}

The early lesion of post-primary tuberculosis is a post obstructive lobular pneumonia that spreads via bronchi for months prior to caseation [1] (Figure 1). MTB antigen in the early post-primary phase was detected by im-munohistochemistry as a brown chromogenic signal within alveolar macrophages (Figure 1A), alveolar pneu-mocytes (Figure 1B), and ciliated bronchiolar epithelial cells (Figure 1C), Alveolar monocytes and macrophag-es are not necessarily foamy in the early post-primary phase and show mild expression of MTB antigen com-pared with the later phases. The mycobacterial antigens were diffusely present throughout the cytoplasm of the cells as is characteristic of secreted antigens rather than the discrete spots characteristic of intact organisms [7]. PDL1 expression was present on alveolar pneumocytes, sloughed bronchiolar epithelial cells, and alveolar monocytes and macrophages (Figure 2A). PD-1 staining was present on lymphocytes primarily in the interstiti-um (Figure 2B). COX-2 expression was evident by immunohistochemistry within reactive alveolar pneumo-cytes (Figure 2C) and bronchiolar epithelial cells (Figure 2D) in the early post-primary phase of human tuberculous pneumonitis. Alveolar macrophages in the early lesion of post-primary tuberculosis stain with CD163, a marker for M2 polarized macrophages (Figure 2E), Finally, fatty acid synthase (FAS) expression was present in the cytoplasmic compartment of reactive alveolar pneumocytes surrounding increasingly foamy alveolar macrophages (Figure 2F). 


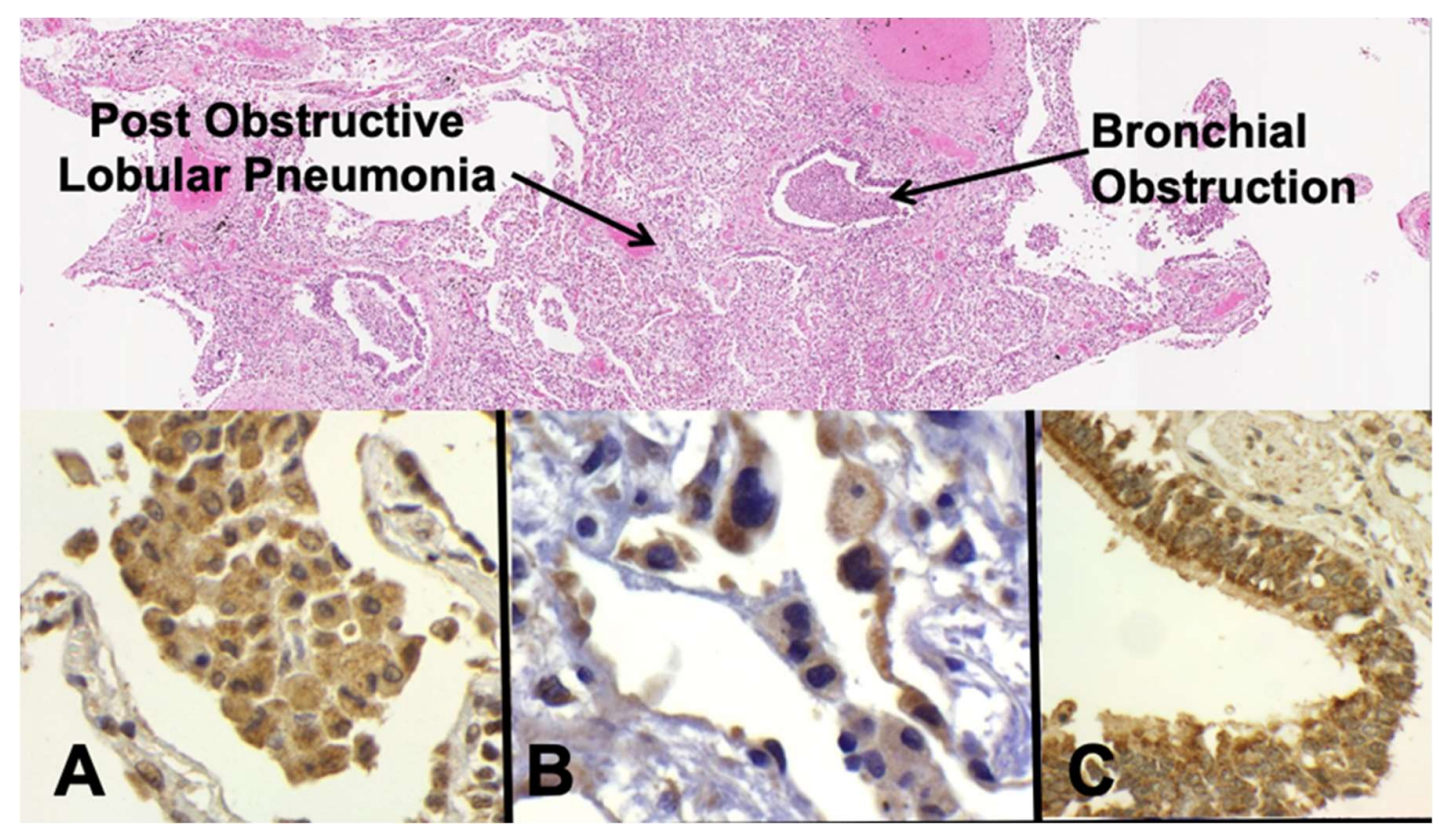

Figure 1. MTB Antigen in the Early Lesion of Post-primary Tuberculosis. Post-primary tuberculosis be-gins as and alveolitis that spreads subclinically as an obstructive lobular pneumonia for 1-2 years before under-going necrosis as caseous pneumonia to initiate clinical post-primary TB. Evidence suggests that MTB uses its secreted antigens to direct these lesions towards cavitation that can transmit infection to new hosts (H\&E stain 20x).

A. Mycobacterial antigens in alveolar macrophages (Immuno stain 600x).B. Mycobacterial antigens alveolar lining cells types 1 and 2 (Immuno stain 600x).C. Mycobacterial antigens in ciliated bronchial cells (Immuno stain 400x). 


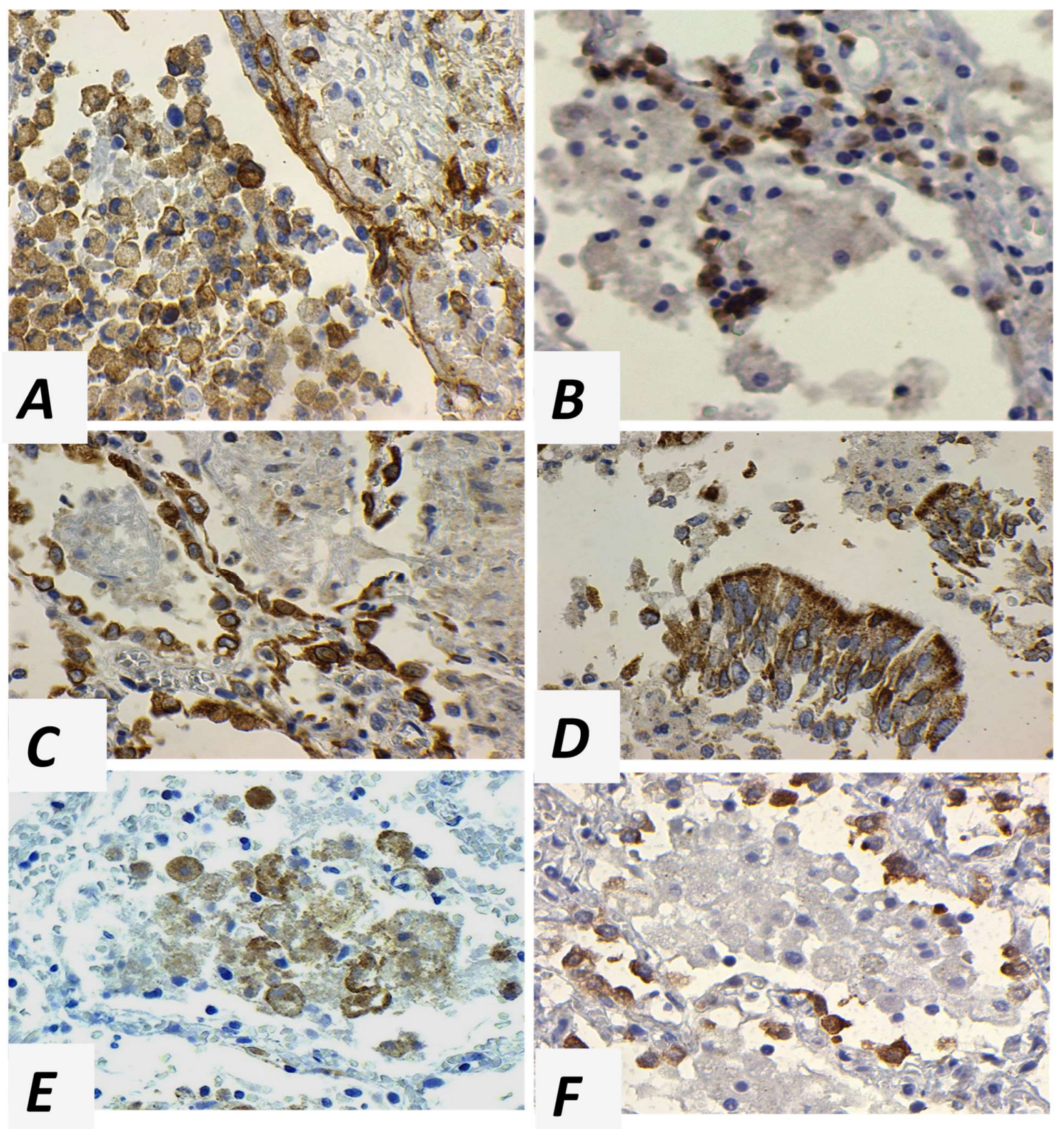

Figure 2. Characterization of the Early Lesion of Post-primary Tuberculosis. Immunostains for PD-L1, PD-1, COX-2, CD163 and FAS provide evidence that MTB through its secreted antigens manipulates the host's responses is the early lesion to develop conditions for a necrotizing reaction sufficient to form a cavity from which it an escape to infect new hosts.

A. PD-L1 expression on alveolar pneumocytes and on alveolar macrophages (600x).B. PD-1 expression on lymphocytes in adjacent alveolar walls (600x).C. COX-2 in reactive alveolar pneumocytes (600x).D. COX-2 in bronchiolar epithelium (600x).E. CD163 staining of alveolar macrophages marking them as M2 cell (400x).Figure 400. x).

\section{Discussion}

The finding of secreted mycobacterial antigens in multiple cell types of the early lesion of PPTB suggests a new synthesis of the pathogenesis of post-primary TB driven by secreted antigens and cord factor (trehalose 6,6' dimycolate). Since MTB is an obligate human parasite, everything that it does has been selected to insure survival and 
transmission among people. MTB secretes at șeppleast 257 proteins into surrounding media that are not necessary for its survival in culture, but are required for virulence in humans [8]. The functions of some se-creted proteins such as ESAT-6 and Ag85 have been studied, but most remain unknown $[8,9]$. Similarly, viru-lent MTB secrete large mounts of trehalose 6,6' dimycolate (TDM or cord factor) an insoluble lipid that re-mains on the surface of the organisms [10]. It is also required for virulence, but not for growth in vitro. It remains on the surface of the organisms and is responsible for the formation of serpentine cords and protects MTB from killing by macrophages [11].

The early lesion of PPTB is an asymptomatic obstructive lobular pneumonia that develops for 1-2 years before onset of symptoms [12]. It is a prolonged asymptomatic accumulation of mycobacterial antigens and host li-pids in alveolar macrophages with little or no inflammation even though there may be intense tuberculous in-flammation elsewhere in the same lung [7]. The process slowly spreads via bronchi in a process known as bronchogenic TB and may be observed as the 'tree-in-bud' sign on CT scans $[5,13]$. The finding of MTB antigens in multiple cells of the early lesion suggests that MTB use these antigens to manipulate diverse components of the host response to asymptomatically produce the components necessary for a sudden and massive necrotiz-ing reaction sufficient to form a cavity large enough to support transmission of infection.

To accomplish this, MTB must evade diverse inflammatory responses while it secretes antigens that manipulate its host. MTB has a remarkable ability to do this. While some tubercle bacilli produce massive tissue damage, especially in the lung, others simultaneously persist in many tissues with no gross evidence of infection [14]. Available evidence suggests that this is mediated by TDM that forms cylindrical micelles and an intercalated bilayer with surfaces composed entirely of trehalose $[15,16]$. We reported evidence that these trehalose sur-faces bind and immobilize water to produce an "invisibility cloak" that facilitates the persistence of MTB in multiple cell types without producing inflammation, even in highly immune individuals [17].

The invisibility cloak of MTB makes it invisible to receptors. Instead of seeing a multitude of pathogen-associated molecular patterns (PAMPs) and epitopes, receptors in cells containing such MTB would see only water that will not move out of the way. Such a mechanism could contribute to the ability of MTB to infect many types of cells and to persist in grossly normal tissues in people with otherwise strong immune responses and severe inflammation and granulomas in other parts of the body. In particular, we propose that this mecha-nism is an important part of developing post-primary TB that is characterized by asymptomatic accumulation of mycobacterial antigens and host lipids in lungs of highly sensitized people. An invisibility cloak would pro-vide MTB with ability to manipulate its environment in at least two ways. First, MTB would not have to de-velop means of neutralizing or evading all of the defenses of many types of cells. Second, MTB could release signaling molecules of its choosing to manipulate the functions of the host for its benefit.

The early lesion of post-primary TB involves asymptomatic coordination of multiple types of cells in the lung for a period of months prior to undergoing necrosis to produce caseation pneumonia that is coughed out to form a cavity or retained to become the focus of post-primary granulomas and fibrocaseous disease. The com-ponents of the early lesion include:

1) Alveolar macrophages that have the M2 phenotype (CD163 Staining)

2) Bronchial obstruction traps alveolar macrophages to produce a post-obstructive lipid pneumonia [18].

3) Increase production of lipids by alveolar lining cells (FAS) that are taken up by macrophages that become increasingly foamy.

4) Accumulation of secreted mycobacterial antigens in the foamy alveolar macrophages.

5) Stimulation of highly sensitized tissue resident T cells (TRM) in adjacent alveolar walls. 
6) Suppression of T cell activity with PD-L1.

These lesions spread via bronchi as an obstructive lobular pneumonia, recognized by CT scans as the 'tree-in-bud' sign, until they either regress or undergo necrosis to become caseous pneumonia that gives rise to cavities and post-primary granulomas.

Our new findings include demonstration of mycobacterial antigen within both the alveolar pneumocytes and detached bronchiolar lining cells with mild expression in associated alveolar monocytes/ macrophages; marked COX-2 expression in reactive alveolar pneumocytes and detached bronchiolar epithelium, the latter potentially leading to obstruction and contributing to lipid pneumonia; expression of fatty acid synthase (FAS) that could contribute fatty acids to the alveolar macrophages; expression of programmed death ligand (PD-L) 1 on the alveolar monocytes/macrophages and on the pneumocytes and bronchiolar epithelial cells; and CD163 expres-sion on the non-foamy monocytes/macrophages in the alveolar intersitium and alveoli.

In previous studies we have reported that the early lesion of post-primary tuberculosis contains foamy alveolar macrophages with the M2 phenotype that marked with CD163, PD-L1, phosphorylated mTOR, insulin-like growth factor-1 receptor (IGF-1R), and human cyclooxygenase 2 (COX-2) [19]. Relatively few CD4+ cells were present, but abundant CD8+, PD-L1 cells were in the alveolar walls, Foamy macrophages stained strong-ly with CD68 and frequently also with the dendritic cell marker DEC-205. T-regulatory cells were also ob-served in the early lesion [20, 21]. These findings indicate that MTB creates a protective microenvironment that accumulates high concentrations of MTB antigens and sensitized T cells that when released lead to necro-sis, cavitation, post-primary granulomas and fibrocaseous disease [21].

Several lines of evidence suggests that the early lesion is an attractive target for vaccines to prevent post-primary tuberculosis. First, many nascent lesions regressed spontaneously. If we knew why it might be possible to induced all to regress. Second, slowly progressive pulmonary tuberculosis in the mouse is a model of the early lesion of postprimary tuberculosis [22]. Several experimental vaccines have been shown to prevent development of these lesions [23-25]. Finally, the ASO/M721E vaccine that was successful in a human clinical trial was designed to induce a strong Th1 macrophage response without an $\mathrm{M} 2$ response [26, 27]. Since a strong Th1/M1 response occurs in people with severe disease, we propose that the protection was provided by suppressing the M2 response and thereby preventing development of the early lesion of post-primary tubercu-losis.

There is also evidence that the early lesion of post-primary TB is an attractive target for host directed therapies [19, 28]. Multiple studies of vitamin D3, metformin, and indomethacin on human specimens in vitro suggest efficacy against the early lesion of PPTB. Mice, guinea pigs and rabbits all develop infections that at certain points appear to be models of human post-primary TB [25]. In multiple studies, effective vaccines and host directed thrapies were associated with lessening of the PPTB like pathology in these models [23, 24, 28-32]. For example, slowly progressive pulmonary TB in immunocompetent mice is characterized by an alveolitis with infected foamy macrophages that have multiple characteristics of the human disease [22]. We demonstrated that inclusion of lactoferrin, with a BCG vaccine in this model induced a sustained reduction in lung patholo-gy, but not numbers of organisms in tissue [23].

Evidence for a mechanism of host directed therapy is provided by studies of fatty acid synthase (FAS). The presence of FAS in alveolar lining cells of the early lesion of PPTB indicates increased synthesis of lipids that is a critical component of these lesions. We demonstrated that inclusion an FAS inhibitor, lactoferrin, with a BCG vaccine in mice induced a sustained reduction in lung pathology, but not numbers of organisms in tissue [23]. Metformin and orlistat are also inhibitors of fatty acid synthase [33-35] and have shown efficacy against TB in preclinical studies of TB [23, 34]. In two separate human trials, metformin treatment was associated with improved control of infection and decreased disease severity $[29,36]$. Vitamin D3 has also been shown to reduce accumulation 
of lipids by macrophages and to produce beneficial effects in patients in a controlled trial of pulmonary tuberculosis [37, 38].

\section{Conclusion}

The finding of secreted mycobacterial antigens in multiple cells of the early lesions together with markers of multiple immune regulatory pathways suggest that these subclinical asymptomatic lesions are actually the ma-jor drivers of clinical post-primary tuberculosis. They serve both to control and direct development of the early lesion and to accumulate antigen targets for necrotizing hypersensitivity reactions. Study of these lesions will remain challenging because of the paucity of informative human tissues that can be used both to study the dis-ease and to validate animal models. Fortunately, new multiplex technologies can measure proteins and nucleic acids on slides with a depth and precision undreamed of a decade ago. Such technologies are needed to finally study these critical lesions of tuberculosis and to develop more effective interventions.

Authors' Contribution: Both authors contributed equally to the writing of the manuscript, and to the review and editing of the final manuscript.

Funding: This research received no external funding.

Conflicts of Interest: The authors declare no conflicts of interest.

\section{References}

1. Hunter RL. Pathology of post primary tuberculosis of the lung: an illustrated critical review. Tuberculosis (Edinb). 2011;91(6):497-509. Epub 2011/07/08. doi: 10.1016/j.tube.2011.03.007. PubMed PMID: 21733755; PubMed Central PMCID: PMC3215852.

2. Rich A. The Pathogenesis of Tuberculosis, Second Edition. Springfield, Illinois: Charles C Thomas; 1951.1028 p.

3. Canetti G. The tubercle bacillus in the pulmonary lesion of man. Histobacteriology and its bearing on the therapy of pulmonary tuberculosis. New York: Springer Publishing Compani Inc.; 1955. 226 p.

4. Medlar EM. The pathogenesis of minimal pulmonary tuberculosis; a study of 1,225 necropsies in cases of sudden and unexpected death. Am Rev Tuberc. 1948;58(6):583-611. Epub 1948/12/01. PubMed PMID: 18099839.

5. Im JG, Itoh H. Tree-in-Bud Pattern of Pulmonary Tuberculosis on Thin-Section CT: Pathological Impli-cations. Korean J Radiol. 2018;19(5):859-65. doi: 10.3348/kjr.2018.19.5.859. PubMed PMID: 30174474; PubMed Central PMCID: PMCPMC6082770.

6. Hunter RL. The Pathogenesis of Tuberculosis-The Koch Phenomenon Reinstated. Pathogens. 2020;9(10):1-25. Epub 2020/10/07. doi: 10.3390/pathogens9100813. PubMed PMID: 33020397.

7. Hunter RL, Actor JK, Hwang SA, Karev V, Jagannath C. Pathogenesis of post primary tuberculosis: immunity and hypersensitivity in the development of cavities. Ann Clin Lab Sci. 2014;44(4):365-87. PubMed PMID: 25361920.

8. Malen H, Berven FS, Fladmark KE, Wiker HG. Comprehensive analysis of exported proteins from My-cobacterium tuberculosis H37Rv. Proteomics. 2007;7(10):1702-18. Epub 2007/04/20. doi: 10.1002/pmic.200600853. PubMed PMID: 17443846.

9. Mustafa T, Leversen NA, Sviland L, Wiker HG. Differential in vivo expression of mycobacterial anti-gens in Mycobacterium tuberculosis infected lungs and lymph node tissues. BMC Infect Dis. 2014;14:535. Epub 2014/10/07. doi: 10.1186/1471-2334-14535. PubMed PMID: 25284264; PubMed Central PMCID: PMC4287340.

10. Behling CA, Bennett B, Takayama K, Hunter RL. Development of a trehalose 6,6'-dimycolate model which explains cord formation by Mycobacterium tuberculosis. Infect Immun. 1993;61(6):2296-303. PubMed PMID: 8500871; PubMed Central PMCID: PMCPMC280848.

11. Indrigo J, Hunter RL, Actor JK. Influence of trehalose 6,6'-dimycolate (TDM) during mycobacterial in-fection of bone marrow macrophages. Microbiology (Reading). 2002;148(Pt 7):1991-8. Epub 2002/07/09. doi: 10.1099/00221287-148-7-1991. PubMed PMID: 12101287.

12. Hunter RL. The Pathogenesis of Tuberculosis: The Early Infiltrate of Post-primary (Adult Pulmonary) Tuberculosis: A Distinct Disease Entity. Front Immunol. 2018;9:2108. Epub 2018/10/05. doi: 10.3389/fimmu.2018.02108. PubMed PMID: 30283448; PubMed Central PMCID: PMCPMC6156532.

13. Medlar EM. Pathogenic concepts of tuberculosis. Am J Medicine. 1950;9:611-22. 
14. Dutta NK, Karakousis PC. Latent tuberculosis infection: myths, models, and molecular mechanisms. Mi-crobiol Mol Biol Rev. 2014;78(3):343-71. Epub 2014/09/04. doi: 10.1128/MMBR.00010-14. PubMed PMID: 25184558; PubMed Central PMCID: PMC4187682.

15. Schabbing RW, Garcia A, Hunter RL. Characterization of the trehalose 6,6'-dimycolate surface mono-layer by scanning tunneling microscopy. Infect Immun. 1994;62(2):754-6. Epub 1994/02/01. doi: 10.1128/iai.62.2.754-756.1994. PubMed PMID: 8300239; PubMed Central PMCID: PMCPMC186174.

16. Hunter RL, Olsen M, Jagannath C, Actor JK. Trehalose 6,6'-dimycolate and lipid in the pathogenesis of caseating granulomas of tuberculosis in mice. Am J Pathol. 2006;168(4):1249-61. doi: 10.2353/ajpath.2006.050848. PubMed PMID: 16565499; PubMed Central PMCID: PMCPMC1606544.

17. Hunter RL, Hwang SA, Jagannath C, Actor JK. Cord factor as an invisibility cloak? A hypothesis for asymptomatic TB persistence. Tuberculosis (Edinb). 2016;101 S2-S8. doi: 10.1016/j.tube.2016.09.023. PubMed PMID: 27743706.

18. Hunter RL. On the pathogenesis of post primary tuberculosis: the role of bronchial obstruction in the pathogenesis of cavities. Tuberculosis (Edinb). 2011;91 Suppl 1:S6-10. Epub 2011/11/16. doi: S1472-9792(11)00173-9 [pii] 10.1016/j.tube.2011.10.003. PubMed PMID: 22082617.

19. Brown RE, Hunter RL, Hwang SA. Morphoproteomic-Guided Host-Directed Therapy for Tuberculosis. Front Immunol. 2017;8:78. doi: 10.3389/fimmu.2017.00078. PubMed PMID: 28210262; PubMed Cen-tral PMCID: PMCPMC5288338.

20. Welsh KJ, Risin SA, Actor JK, Hunter RL. Immunopathology of postprimary tuberculosis: increased T-regulatory cells and DEC-205-positive foamy macrophages in cavitary lesions. Clin Dev Immunol. 2011;2011:307631. Epub 2011/01/05. doi: 10.1155/2011/307631. PubMed PMID: 21197439; PubMed Central PMCID: PMC3010642.

21. Hwang SA, Ali Y, Fedotova E, Hunter RL, Brown RE. Morphoproteomics Identifies the Foamy Alveo-lar Macrophage as an M2 Phenotype with PD-L1 Expression in the Early Lesion of Post-Primary Tuber-culosis: Implications for Host Immune Surveillance and Therapy. Ann Clin Lab Sci. 2020;50(4):429-38. Epub 2020/08/23. PubMed PMID: 32826237.

22. Hunter RL, Jagannath C, Actor JK. Pathology of postprimary tuberculosis in humans and mice: contra-diction of long-held beliefs. Tuberculosis (Edinb). 2007;87(4):267-78. Epub 2007/03/21. doi: S1472-9792(06)00120-X [pii] 10.1016/j.tube.2006.11.003. PubMed PMID: 17369095.

23. Hwang SA, Welsh KJ, Kruzel ML, Actor JK. Lactoferrin Augmentation of the BCG Vaccine Leads to Increased Pulmonary Integrity. Tuberc Res Treat. 2011;2011:835410. doi: 10.1155/2011/835410. Pub-Med PMID: 22567270; PubMed Central PMCID: PMCPMC3335707.

24. Liang Y, Bai X, Zhang J, Song J, Yang Y, Yu Q, et al. Ag85A/ESAT-6 chimeric DNA vaccine induces an adverse response in tuberculosis-infected mice. Mol Med Rep. 2016;14(2):1146-52. Epub 2016/06/10. doi: 10.3892/mmr.2016.5364. PubMed PMID: 27279275; PubMed Central PMCID: PMCPMC4940052.

25. Hunter R, Actor J. The pathogenesis of post-primary tuberculosis. A game changer for vaccine develop-ment. Tuberculosis (Edinb). 2019;116S:S114-S7. PubMed PMID: 31076321; PubMed Central PMCID: PMCPMC6626673.

26. Van Der Meeren O, Hatherill M, Nduba V, Wilkinson RJ, Muyoyeta M, Van Brakel E, et al. Phase 2b Controlled Trial of M72/AS01E Vaccine to Prevent Tuberculosis. N Engl J Med. 2018;379(17):1621-34. Epub 2018/10/04. doi: 10.1056/NEJMoa1803484. PubMed PMID: 30280651; PubMed Central PMCID: PMCPMC6151253.

27. Al-Attiyah R, Mustafa AS, Abal AT, El-Shamy AS, Dalemans W, Skeiky YA. In vitro cellular immune responses to complex and newly defined recombinant antigens of Mycobacterium tuberculosis. Clin Exp Immunol. 2004;138(1):139-44. doi: 10.1111/j.1365-2249.2004.02609.x. PubMed PMID: 15373916; PubMed Central PMCID: PMCPMC1809193.

28. Shim D, Kim H, Shin SJ. Mycobacterium tuberculosis Infection-Driven Foamy Macrophages and Their Implications in Tuberculosis Control as Targets for Host-Directed Therapy. Front Immunol. 2020;11:910. Epub 2020/06/02. doi: 10.3389/fimmu.2020.00910. PubMed PMID: 32477367; PubMed Central PMCID: PMCPMC7235167.

29. Singhal A, Jie L, Kumar P, Hong GS, Leow MK, Paleja B, et al. Metformin as adjunct antituberculosis therapy. Sci Transl Med. 2014;6(263):263ra159. Epub 2014/11/21. doi: 10.1126/scitranslmed.3009885. PubMed PMID: 25411472.

30. Okada M, Kita Y, Nakajima T, Kanamaru N, Hashimoto S, Nagasawa T, et al. Novel prophylactic vac-cine using a primeboost method and hemagglutinating virus of Japan-envelope against tuberculosis. Clin Dev Immunol. 2011;2011:549281. Epub 2011/03/26. doi: 10.1155/2011/549281. PubMed PMID: 21437226; PubMed Central PMCID: PMCPMC3061297.

31. Maurya SK, Aqdas M, Das DK, Singh S, Nadeem S, Kaur G, et al. A multiple T cell epitope comprising DNA vaccine boosts the protective efficacy of Bacillus Calmette-Guerin (BCG) against Mycobacterium tuberculosis. BMC Infect Dis. 2020;20(1):677. Epub 2020/09/19. doi: 10.1186/s12879-020-05372-1. PubMed PMID: 32942991; PubMed Central PMCID: PMCPMC7495405.

32. Doimo NT, Zarate-Blades CR, Rodrigues RF, Tefe-Silva C, Trotte MN, Souza PR, et al. Immunotherapy of tuberculosis with Mycobacterium leprae Hsp65 as a DNA vaccine triggers cross-reactive antibodies against mammalian Hsp60 but not pathological autoimmunity. Hum Vaccin Immunother. 2014;10(5):1238-43. Epub 2014/03/13. doi: 10.4161/hv.28249. PubMed PMID: 24607935; PubMed Central PMCID: PMCPMC4896578. 
33. Kridel SJ, Axelrod F, Rozenkrantz N, Smith JW. Orlistat is a novel inhibitor of fatty acid synthase with antitumor activity. Cancer Res. 2004;64(6):2070-5. Epub 2004/03/18. doi: 10.1158/0008-5472.can-03-3645. PubMed PMID: 15026345.

34. Majumdar A, Wankhade G, Kamble PD, Harinath BC. Effect of HIV protease inhibitors and Orlistat on mycobacterial ES-31 serine protease, a potential drug target in Mycobacterium tuberculosis. Indian J Tu-berc. 2011;58(1):4-10. Epub 2011/03/30. PubMed PMID: 21442799.

35. Wahdan-Alaswad RS, Cochrane DR, Spoelstra NS, Howe EN, Edgerton SM, Anderson SM, et al. Met-formin-induced killing of triple-negative breast cancer cells is mediated by reduction in fatty acid syn-thase via miRNA-193b. Horm Cancer. 2014;5(6):374-89. Epub 2014/09/13. doi: 10.1007/s12672-014-0188-8. PubMed PMID: 25213330; PubMed Central PMCID: PMCPMC4570735.

36. Restrepo BI. Metformin: Candidate host-directed therapy for tuberculosis in diabetes and non-diabetes patients. Tuberculosis (Edinb). 2016;101S:S69-S72. Epub 2016/10/11. doi: 10.1016/j.tube.2016.09.008. PubMed PMID: 27720378.

37. Salamon H, Bruiners N, Lakehal K, Shi L, Ravi J, Yamaguchi KD, et al. Cutting edge: Vitamin D regu-lates lipid metabolism in Mycobacterium tuberculosis infection. J Immunol. 2014;193(1):30-4. Epub 2014/06/06. doi: 10.4049/jimmunol.1400736. PubMed PMID: 24899504; PubMed Central PMCID: PMCPMC4073889.

38. Mily A, Rekha RS, Kamal SM, Arifuzzaman AS, Rahim Z, Khan L, et al. Significant Effects of Oral Phenylbutyrate and Vitamin D3 Adjunctive Therapy in Pulmonary Tuberculosis: A Randomized Con-trolled Trial. PLoS One. 2015;10(9):e0138340. Epub 2015/09/24. doi: 10.1371/journal.pone.0138340. PubMed PMID: 26394045; PubMed Central PMCID: PMCPMC4578887. 